\title{
“Multi-Split VVVF” System for Electric Submersible Pump on Extraction Flat Roof on the Sea
}

XIE Wei ${ }^{1, a}$, CHEN Lei ${ }^{2, b}$, WEI Honggang ${ }^{1, c}$, GUO Lei ${ }^{3, d}$, ZHANG Shengli ${ }^{1, e}$, LIANG Tairan ${ }^{2, f}$, CHEN Jian ${ }^{4, g}$, LI Yaming ${ }^{5, h}$

${ }^{1}$ Petroleum Production Engineering Research Institute of Huabei Oilfield Company, Renqiu, 062552, China

${ }^{2}$ Exploration and Development Research institute of Huabei Oilfield Company, Renqiu, 062552, China

${ }^{3}$ Jidong Oilfield Company Development Department,Tangshan,063000,China

${ }^{4}$ Sulige Project Department of Huabei Petroleum Administration Bureau,Sulige, 017399,China

${ }^{5}$ The First Exploitation Factory of Huabei Oilfield Company,Renqiu,062552,China

acyy_xiew@petrochina.com.cn, ${ }^{\text {b } y j y \_c h e n l e i @ p e t r o c h i n a . c o m . c n, ~}{ }^{c}$ cyy_weihg@petrochina.com.cn, dnpqgl@petrochiana.com.cn, ${ }^{\mathrm{e}} \mathrm{cyy}$ _zzhangsl@petrochina.com.cn, ${ }^{\mathrm{f}} \mathrm{yjy}$ _ttr@petrochina.com.cn, ghbytslg_cj@petrochina.com.cn, ${ }^{\text {h}} c y 1$ lyam@petrochina.com.cn

Keywords: VVVF; PLC; field bus; electric submersible pump; synchronization switch Abstract: This Paper introduces the usage of an automatic system, which uses one VVVF controls many electric submersible pumps. "Multi-Split VVVF" system means that only use one (or a few) frequency converter using multiple PLC components network control system. The system can start and stop control of multiple motors, protect the motor and frequency conversion and power frequency automatic switching. Advantages are small space, less investment funds. The whole system is in the leading position in the country. The degree of automation is relatively high, has gradually meet the requirements of an unmanned platform. There is a very broad application prospects.

\section{Introduction to "Multi-Split VVVF" system}

In today's industrial production, the frequency converter has been widely used. We use frequency converter driven electric submersible pump (ESP) in the offshore platform, which makes a hard start into a soft start. Frequency converter used increased the service life of the ESP, and in some complex well conditions can also smoothly start-up of the ESP. At the same time, the frequency converter can also free control of ESP speed and energy saving. Therefore, we use the ESP according to the characteristics of offshore platform, design of the "Multi-Split VVVF" system.

"Multi-Split VVVF" system means that in some special conditions (such as space, funds, etc.) only use one (or a few) frequency converter using multiple PLC components network control system. The system can start and stop control of multiple motors, protect the motor and frequency conversion and power frequency automatic switching. Advantages are small space, less investment funds. The disadvantage is an ESP cannot be used alone a frequency converter. Due to adapt to multiple different specifications of ESP, frequency converter protection parameter setting is 
relatively loose, easy to cause the motor cannot be protected. These conditions must be solved by other means.

We use "Multi-Split VVVF" system on the offshore platform without the use of one to one frequency control system is due to the small area of platform, ESP power is relatively large and the size of frequency conversion is large, resulting in no space to install. According to the situation, we chose Germany SIEMENS frequency conversion and Admiralty Moeller PLC system.

\section{The basic principle of "Multi-Split VVVF" system}

"Multi-Split VVVF" system is the frequency converter, which occupies a very important position in the whole system, so the type of frequency converter is directly related to the stability of the whole system. There are two good solutions: the first solution is the direct use of high voltage frequency converter; the second solution is the use of low-voltage frequency converter with the method of boosting transformer. The use of high voltage frequency converter solution its equipment is less, easy to use, easy to get a good control effect, but the cost is expensive; The use of low voltage frequency converter solution its cost is low, if the design is reasonable, also can achieve a good control effect. After careful comparison, we chose two SIEMENS 6sE71 cabinets of low-voltage frequency converter.

As Fig.1show, the principle of "Multi-Split VVVF" system is as follows: First, the voltage on the offshore platform is $380 \mathrm{~V}$, after the rectifier and boost transformer output 660-690V voltage; Second, through the reactor into the low-voltage frequency converter, its output through the HVM filter; Third, after a three tap frequency step-up transformer output, through the different ratio by high voltage switch cabinet shift output different voltage, which were suitable for different specifications of the ESP. Because the low voltage frequency converter output is up to $690 \mathrm{~V}$, and the voltage span of the platform is very large $(800 \mathrm{~V}-2400 \mathrm{~V})$. Only one output ratio cannot meet the requirements, so we design a three tap variable frequency boost transformer. It has three gears ratio, so as to meet the requirements of the platform.

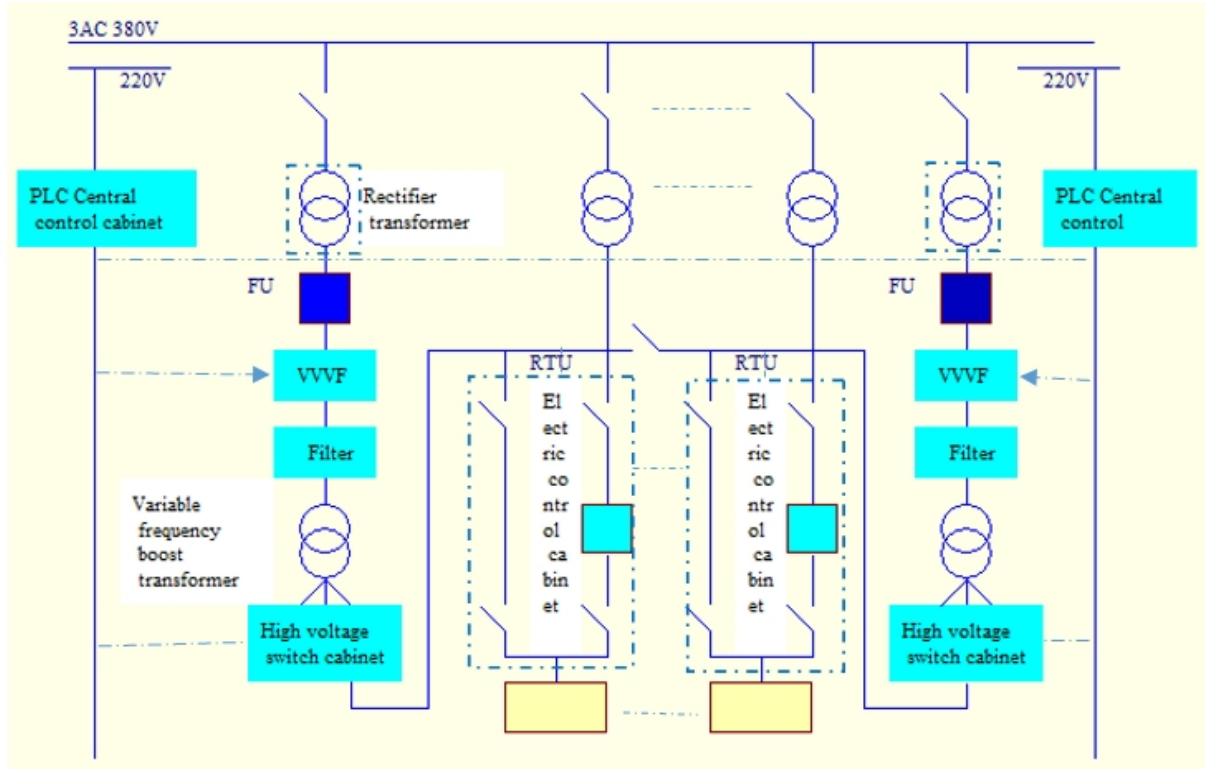

Fig.1 System principle diagram

We designed two sets of the same system A and B, each of which controls one frequency converter respectively. Under normal working conditions, system A and B respective management half of the ESP; when failures occur in a system, all the electrical submersible pump (ESP) taken 
over by another set of system, to standby for each other. Two systems can be started at the same time under normal circumstances; when the system works, the A and B system have the function of software interlock and hardware interlock to ensure the safe operation of the system.Fig.2shows SUCONET Bus control principle.

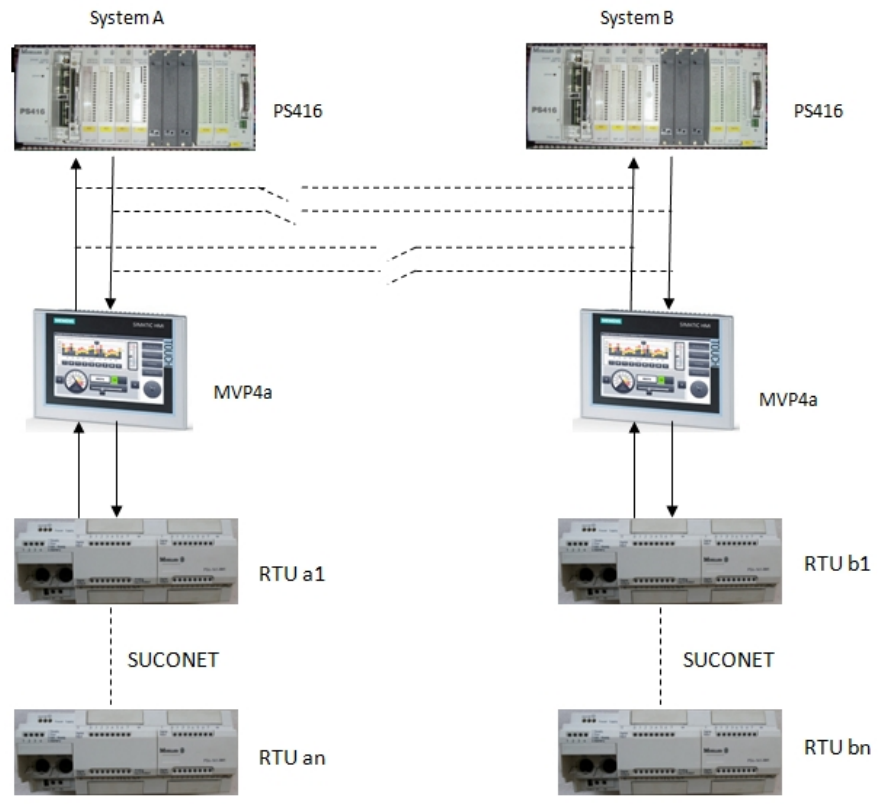

Fig.2 SUCONET Bus control principle diagram

\section{Design of control circuit of "Multi-Split VVVF" system}

The whole system of control circuit requirements is relatively high. In addition to the stable operation, but also need to have perfect control, monitoring, limited data storage, printing, software protection, malfunction alarm, network communication, simple operation and other function. In order to achieve the above requirements, taking into account the compatibility and stability, we chose a complete set of German Muller products: We chose the MV4 touch screen as the display system; select the large PLC system as the central control part; select Ps4-141-MMI (or ps4-151-MMI) as RTU.

The whole system uses three kinds of network protocols: SUCONET K、PROFIBUS DP、 MODBUS. SUCONETK protocol is a kind of field bus standard of German Muller. Through this protocol MV4 and RTU connected and communicated with the central PLC. We use two SUCONET master stations, i.e. CPU and a SUCONET card - PS4 416-NET-400. This increases the system redundancy (theoretical maximum driven 60 slave station), greatly accelerate the reaction speed of the CPU, but also increases the stability of the system. Due to the special requirements of offshore oil production platform, we also use two sets of systems in the software system. Under normal operation, system A and B each managed n ESP, they can work independently, allow the simultaneous launch of two ESP. When a system fault occurs, can toggle switch A or B, normal system managed all of the ESP, where A and B switch interlock.

PROFIBUS protocol is the field bus standard developed by SIEMENS Corporation. Wide used in all kinds of SIEMENS products, including its frequency converter. Therefore, we use this protocol to establish the connection between the frequency converter and the central PLC, so as to achieve the purpose of automatic control of the frequency converter. In order to establish the communication between the system and the platform, we chose the MODBUS field bus, which can communicate with the host computer, data upload and remote monitoring platform. To achieve an unmanned platform, A and B system can standby each other. 
To start the ESP as an example to illustrate the working process: First, in mv4 (or on the remote control platform of the host computer), we chose to start an ESP, a command is transmitted to the central PLC, after confirm the command without error, PLC immediately check the relevant hardware state, to confirm the normal and send commands to the corresponding RTU ready to start the pump; Second, automatic calculated parameters, send electrical submersible pump voltage data to frequency converter, ESP is determined by the voltage, overload current and high voltage switch cabinet ratio and make them connected; Third, send the starting command of the ESP to the RTU, after confirming start the frequency converter. In the process of starting the frequency converter, the frequency of the converter can be artificially adjusted. Confirm the ESP in normal operation, you can switch convenient manual selection switch button, switch variable frequency / power frequency. After the switch is successful, return to the initial state. In the starting process of the frequency converter, the ESP may be encountered in the overload situation, at this time the pump is protected by the central PLC. When the current is greater than the rated current and less than 2 times the rated current, the central PLC control frequency converter automatic frequency drop, until not overload so far, if more than 30 seconds is still overload, then the emergency stop frequency converter; When the current is more than 2 times the rated current, the frequency converter will be stopped immediately. We should pay attention to that the operation of the ESP in less than $30 \mathrm{~Hz}$ cannot be a long time, so at the start of the pump must be monitoring frequency converter operating frequency. The Specific process is completed by the detection module. Flow chart is shown in Fig.3.

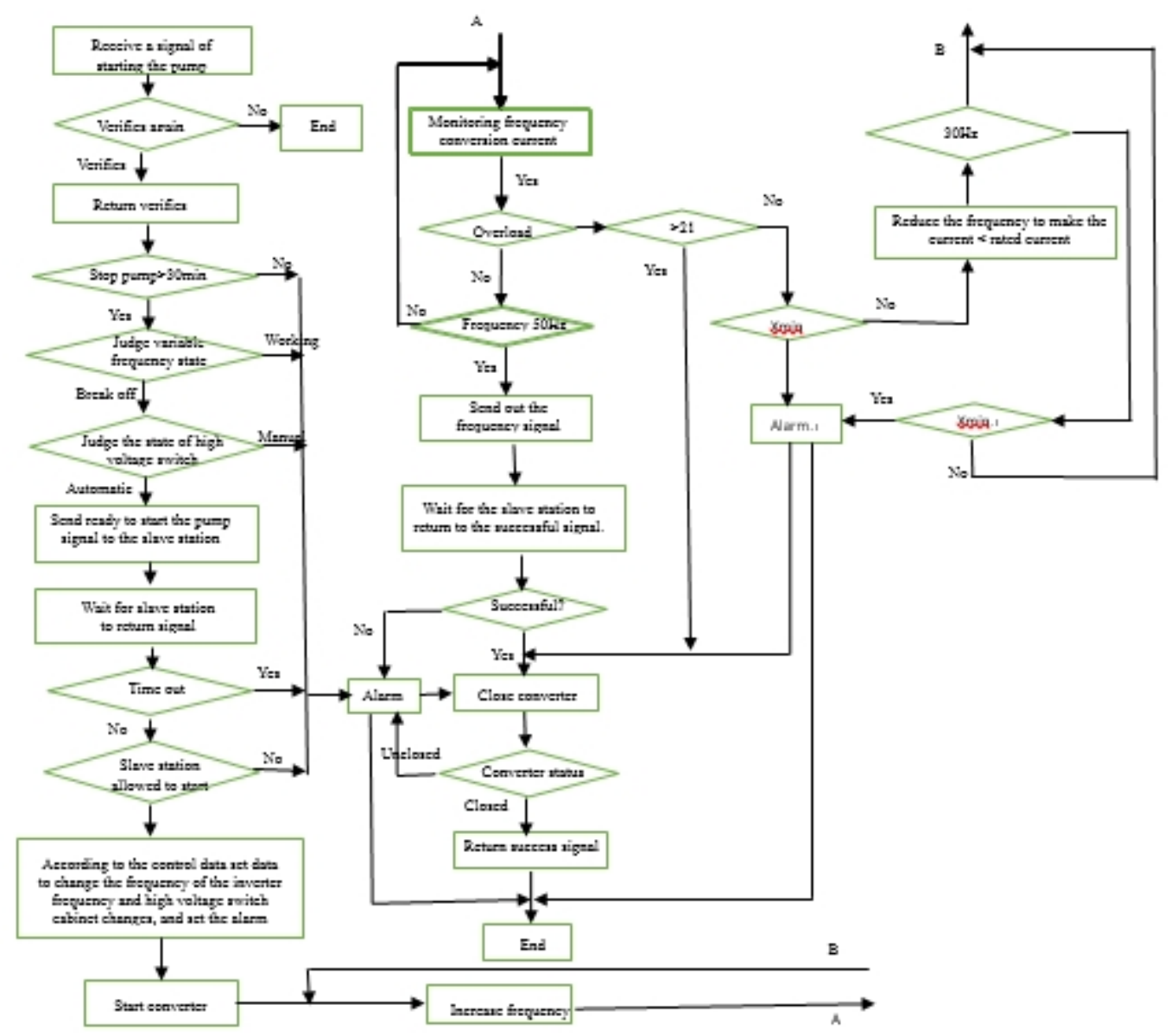

Fig.3 Start the electric submersible pump flow chart

In the process of starting ESP allows the frequency converter to adjust the frequency from 30 to $60 \mathrm{~Hz}$, and a real time frequency-current curve is shown to observe the well condition, and the 
artificial selection switch is selected in the case of well recognized well condition (the switching process is completed by RTU, completely automatic.).

In order to prevent the fault of the automatic control part, that is SUCONET network failure, resulting in the system paralysis, the system in a single RTU operation under normal circumstances, but also increased the RTU manual soft start function. At this time converter operating parameters need to manually set into, manual calculation of ratio of high voltage switch cabinet and the high-voltage switch to the appropriate location. Manual starting frequency converter to $50 \mathrm{~Hz}$ (cannot adjust the frequency), RTU will automatically complete the frequency conversion / power frequency switching. This can only serve to reduce the starting current of the ESP, not free to control the frequency of the frequency converter and the switching time of frequency conversion / power frequency.

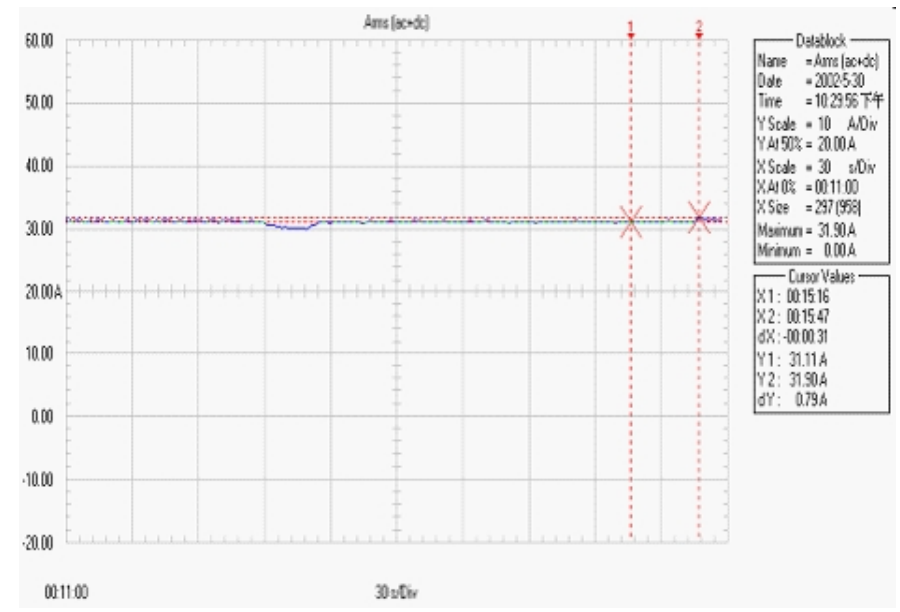

Fig.4 Switching of D8 well in Qinhuangdao 32-6D platform

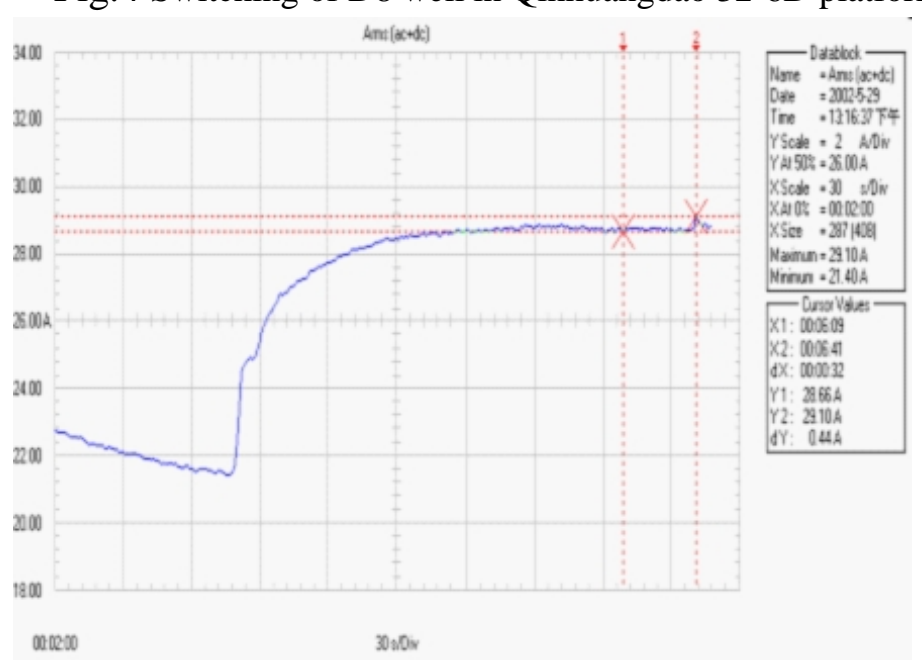

Fig.5Switching of D14 well in Qinhuangdao 32-6D platform

\section{About frequency conversion / power frequency switching}

The traditional way to switch is to stop the frequency converter, and then connected to the power frequency. The advantage of this method is simple, but at the same time as the motor may still rotate, causing the current is too large to switch to failure or cause motor damage, cannot achieve the purpose of real protection. Analysis of the reasons, the frequency conversion / power frequency switching current is too large because of this time frequency conversion and frequency of the phase is not consistent. Therefore, we design phase and phase sequence comparator, by 
comparing the phase of frequency conversion and power frequency, the same time of the phase is judged and the fast switching is carried out by RTU. Through the program of RTU adjustment, considering the relay connectivity and release time, switching time (the difference between frequency conversion released time and the power frequency connected time) in $10-15 \mathrm{~ms}$, so switching current can guarantee within 1.2 times the rated current, realize synchronous switching. RTU program was joined the procedures to prevent the merger network.

\section{Field application}

This system has been used in the SuiZhong 36-1H of the CNOOC Tianjin branch and Qinhuangdao 32-6D, E, F, run smoothly and the effect is good. After testing the switching current is controlled within 1.2 times, there are some wells because of the relay and the air switch response time is different, resulting in a relatively large switching current (rated current 1.2-1.5 times), by adjusting the parameters of the switching time are significantly improved. Fig. 4 and Fig.5 is the use of small FLUKE43 oscilloscope recording of the switching current, sampling frequency $25 \mathrm{MHz}$, the previous point is to take the comparison point, the latter point is the actual switching point current.

\section{Conclusion}

The whole system, both the hardware and the software, is in the leading position in the country, especially after more than a year of operation and improvement, the degree of automation is relatively high, has gradually meet the requirements of an unmanned platform. Suitable for the automation of the new platform and the transformation of the old platform automation, there is a very broad application prospects.

Note 1: The motor in the state of power frequency $50 \mathrm{~Hz}$ direct start, fast speed, high impact current. Frequent hard start will damage the motor and impact the pump life. In the well, sometimes ESP was difficult to start caused by heavy oil, even unable to start.

Note 2: When the motor starts from slow to fast to speed up the process of the rated speed, that is, the motor drives the motor from $0 \mathrm{~Hz}, 1 \mathrm{~Hz}, 2 \mathrm{~Hz} \ldots$. Slowly start to $50 \mathrm{~Hz}$ stable operation. Soft start without impact current, it is beneficial to extend the life of the pump.

Note 3: When the frequency, amplitude and phase of the frequency conversion power supply are in accordance with the power frequency, by means of electronic devices and control software, the motor operating in the frequency conversion state is converted to the power frequency state. It is different from the grid connected switching. The grid connected switching is to take the frequency conversion and power frequency power supply together with the motor for a period of time, and then to switch, synchronous switching frequency conversion power supply and power frequency power supply is not connected.

\section{Reference:}

[1]SIEMENS SIMOVERT MASTERDRIVES vector control, SIEMENS electric drive Co., Ltd., December 2006.

[2]MOELLER Special Catalogue Automation Products, Moeller GmbH, March 2003.

[3]PWM type frequency converter and soft switch control method, Machinery Industry Press, 2001 